\title{
Frequency-swept ultrasound-modulated optical tomography of scattering media
}

\author{
Lihong V. Wang and Geng Ku \\ Optical Imaging Laboratory, Biomedical Engineering Program, Texas A\&M University, College Station, Texas 77843-3120
}

Received January 26, 1998

\begin{abstract}
A novel frequency-swept ultrasound-modulated optical tomography technique was developed to image scattering media. A frequency-swept ultrasonic wave was used to modulate the laser light passing through a scattering medium. The modulated light was received by an optical detector and was heterodyned with a reference frequency sweep. The heterodyned signal was recorded in the time domain and was then analyzed in the frequency domain to yield a one-dimensional image along the ultrasonic axis. Multiple one-dimensional images obtained at various positions perpendicular to the ultrasonic axis were combined to yield a two-dimensional tomographic image of the medium. (c) 1998 Optical Society of America
\end{abstract}

OCIS codes: $110.7050,110.6960,110.7170$.

Optical tomography of scattering media including biological tissues is a growing research area and has the potential to provide new imaging tools for medical applications. ${ }^{1}$ The noninvasive optical imaging techniques use nonionizing radiation and can provide functional information about biological tissues in vivo. All the optical techniques have to overcome the problem caused by scattering of light in the media to recover imaging information from light that has passed through the media. Sophisticated inverse algorithms are typically applied in time- and frequency-domain techniques to reconstruct images of scattering media. Hybrid techniques that combine relatively transparent radiation with strongly scattering optical radiation are being developed as well. ${ }^{2-6}$

We report a novel imaging technique: frequencyswept ultrasound-modulated optical tomography of scattering media. In this technique a frequencyswept (chirped) ultrasonic wave was used to encode the transmitted laser light. Decoding the transmitted light in the frequency domain allowed us to image objects buried inside the scattering media. A onedimensional (1D) image along the ultrasonic axis was obtained with a single time-domain signal.

A block diagram of the experimental setup is shown in Fig. 1. A function generator produced a frequencyswept signal, which then was amplified in power and amplitude by a power amplifier and a transformer, respectively. The instantaneous frequency of the frequency sweep was

$$
f_{s}(t)=a_{s}+b t
$$

where $a_{s}$ was the starting frequency, $b$ was the sweep rate, and $t$ was the time. In our experiment the frequency sweep was between 7.0 and $10.0 \mathrm{MHz}$ with a sweep rate of $297 \mathrm{MHz} / \mathrm{s}$. The amplified signal was applied to an ultrasonic transducer. The ultrasonic wave propagated vertically into a scattering medium, which was contained in a glass tank. An ultrasound absorber was placed at the bottom of the tank to avoid ultrasonic reflection from the water-glass interface.
A $\mathrm{He}-\mathrm{Ne}$ laser beam at the $633-\mathrm{nm}$ wavelength, after being broadened to $15 \mathrm{~mm}$, illuminated the scattering medium perpendicularly to the ultrasonic column. The laser light was modulated by the ultrasonic column. The instantaneous frequency of ultrasonic modulation along the column was

$$
f_{s}(t, z)=a_{s}+b\left(t-\frac{z-z_{0}}{v_{s}}\right)
$$

where $z$ was the ultrasonic axis, $z_{0}$ was the $z$ coordinate of the point of ultrasonic emission in the ultrasonic transducer, and $v_{s}$ was the speed of sound in the medium. Equation (2) was valid for $t \geq\left(z-z_{0}\right) / v_{s}$. A photomultiplier tube (PMT) collected some transmitted light and converted the optical power into an electric signal. The gain of the PMT was modulated for heterodyne detection by a reference modulation signal produced from another function generator. The reference modulation signal was also frequency swept and had an instantaneous frequency

$$
f_{r}(t)=a_{r}+b t
$$

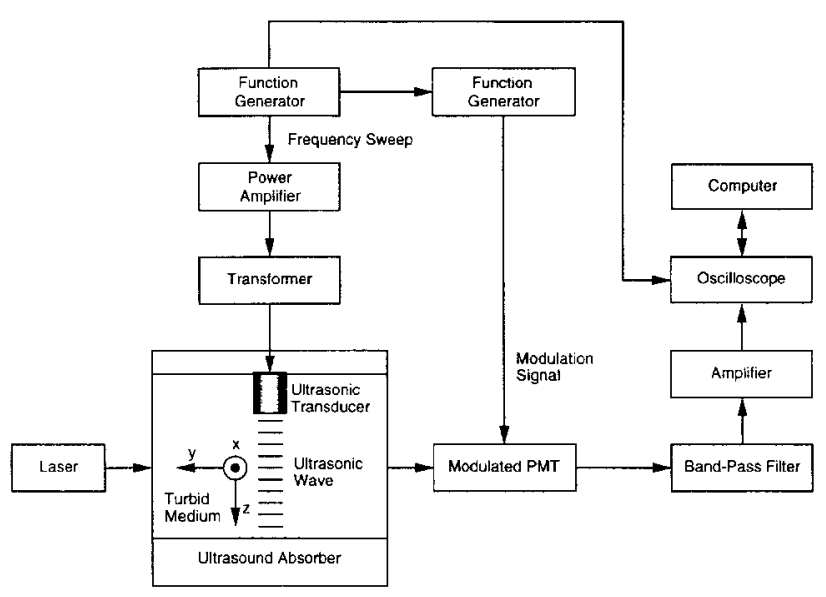

Fig. 1. Block diagram of the experimental setup. 
where the starting frequency $a_{r}$ of the reference modulation signal was different from that of the signal for the ultrasonic transducer.

The frequency of the heterodyned signal contributed from the ultrasonic modulation at $z$ was

$$
f_{h}(t, z)=\left|f_{s}(t, z)-f_{r}(t)\right|=\left|a_{s}-a_{r}-\frac{b\left(z-z_{0}\right)}{v_{s}}\right|,
$$

which was independent of time $t$.

The electronically heterodyned signal at the output of the PMT was bandpass filtered and was then amplified by use of a filter and an amplifier, respectively. The bandwidth of the filter was determined by the desired range of $z$ to be imaged (zone of detection) as follows:

$$
\Delta f_{h}=\frac{b}{v_{s}} \Delta z
$$

where $\Delta z$ was the range of $z$ to be detected. The amplified signal was recorded by a digital oscilloscope and was then transferred to a computer for postprocessing.

An object made of rubber was buried in the middle plane of the tank. The thickness of the tank along the laser beam was $17 \mathrm{~cm}$. The object was translated in the tank along the $x$ axis perpendicularly to both the laser beam and the ultrasonic column. A timedomain signal was recorded at each stop. A fast Fourier transform was used to compute the spectra of the recorded time-domain signals on a computer.

The frequency spectra yielded imaging information for the zone of detection. A frequency in the spectra corresponded to the difference between the instantaneous ultrasonic frequency at an ultrasonic axial position and the instantaneous frequency of the reference modulation signal [Eq. (4)]. By use of Eq. (4) the frequency spectra were converted into 1D images of the scattering medium along the ultrasonic axis ( $z$ axis). The imaging contrast was caused by spatial variation in the optical and acoustic properties.

Two sample frequency spectra are shown in Fig. 2. Figure 2(A) shows a spectrum when the object was far from the ultrasonic axis. Figure 2(B) shows a spectrum when the object blocked part of the laser beam along the ultrasonic axis. The scattering coefficient and the anisotropy of the scattering medium were $0.16 \mathrm{~cm}^{-1}$ and 0.73 , respectively. Some of the frequency components, corresponding to the location of the object, disappeared as indicated in Fig. 2(B). This figure demonstrates the one-to-one correspondence between the heterodyned frequency and the position along the ultrasonic axis.

Multiple 1D spectra were acquired while the buried object was scanned horizontally along the $x$ axis with a step size of $1 \mathrm{~mm}$. The first spectrum, which was taken when the object was far from the ultrasonic axis, was used as a reference spectrum. The spectral intensity of the subsequent spectra was divided by that of the reference spectrum to yield a relative spectral intensity. The 1D relative spectra at the various $x$ positions were combined to form a two-dimensional (2D) image of the medium.

Figure 3 compares two 2D images of the buried object taken in clear water and in a scattering

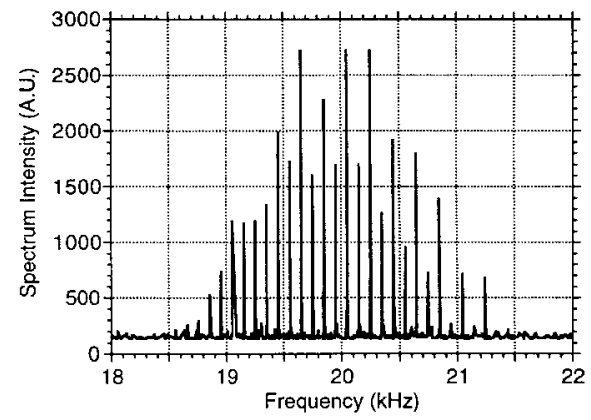

(A)

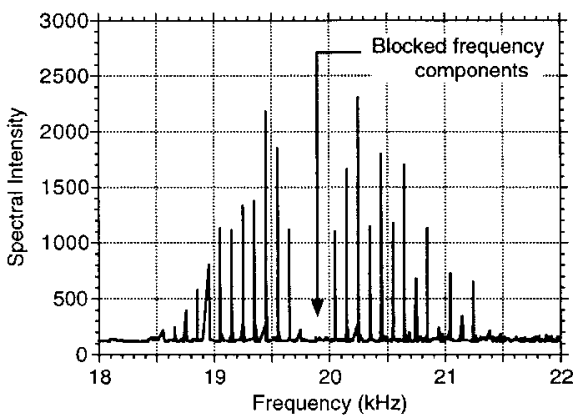

(B)

Fig. 2. Frequency spectrum of the heterodyned frequencyswept ultrasound-modulated optical signal when the object was (A) far from the ultrasonic axis and (B) on the ultrasonic axis.

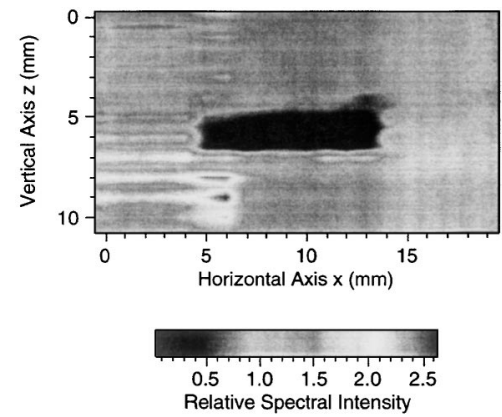

(A)

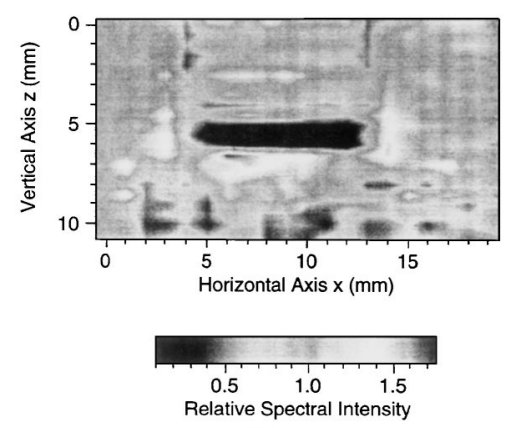

(B)

Fig. 3. 2D tomographic image of the object buried (A) in clear water and (B) in a scattering medium. The object was $8.4 \mathrm{~mm} \times 1.6 \mathrm{~mm}$ in size. 
(A)

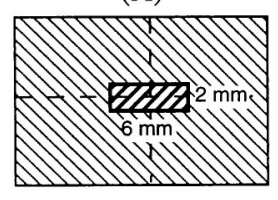

(B)

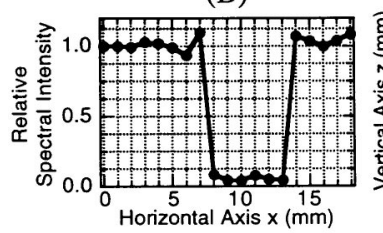

(C)

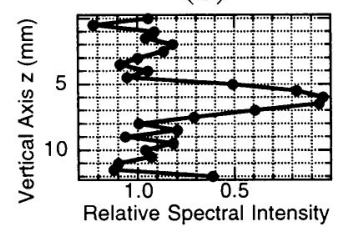

(D)

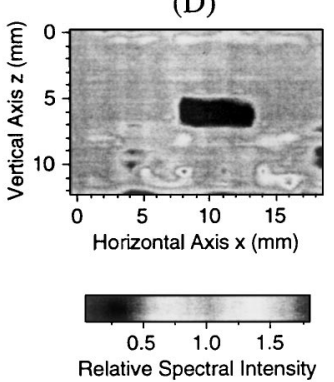

Fig. 4. (A) Cross-sectional view of the object buried in a scattering medium. (B) 1D image of the scattering medium horizontally across the center of the object as indicated by the horizontal dashed line in (A). (C) 1D image of the scattering medium vertically across the center of the object as indicated by the vertical dashed line in (A). (D) 2D tomographic image of the object buried in a scattering medium. The object was $6 \mathrm{~mm} \times 2 \mathrm{~mm}$ in size.

medium. The buried object was optically opaque and relatively acoustically transparent. The optical properties of the scattering medium were the same as those in Fig. 2. The object was clearly visible in both images. The relative spectra density was greater than unity in some of the relative spectra, probably because of the artifacts of digital fast Fourier transform, experimental interference, and system noise.

Another 2D image of the object buried in a scattering medium is shown in Fig. 4. The corresponding 1D images in both the $x$ and the $z$ directions were examined in detail, as shown in Figs. 4(B) and 4(C), respectively. The scattering coefficient and the anisotropy of the scattering medium were $0.21 \mathrm{~cm}^{-1}$ and 0.73 , respectively. As can be seen, the resolution in both the $x$ and the $z$ axes was approximately $1 \mathrm{~mm}$. The $z$-axis resolution was limited by the ultrasonic sweep parameters, and the $x$-axis resolution was limited by the ultrasonic focal diameter $(1.2 \mathrm{~mm})$.

This technique is currently limited to ballistic imaging and is being extended for diffuse-light imaging.
Because ultrasonic modulation of diffuse light with single-frequency ultrasound has been observed, ${ }^{3,4,7,8}$ ultrasonic modulation of diffuse light by use of frequencyswept ultrasound should be detectable as well. The current detection system is being improved to facilitate observation of ultrasonic modulation of diffuse light by frequency-swept ultrasound. The signal-to-noise ratio of the system could not be improved by arbitrary narrowing of the bandwidth of the detection system because the bandwidth was proportional to the sweep rate of the frequency sweep and the zone length of detection, as indicated in Eq. (5). However, a slow frequency sweep and a modest zone of detection can be used to narrow the bandwidth. Note that the speed of sound is relatively constant in a medium and cannot be varied to improve the signal-to-noise ratio.

In summary, we have experimentally proved the concept of frequency-swept ultrasound-modulated optical tomography. A 1D image of a scattering medium along the ultrasonic axis can be obtained with a single time-domain signal. Therefore the image acquisition time can be shortened significantly compared with that for single-frequency ultrasound-modulated optical tomography. The imaging resolution is adjustable with the bandwidth of the frequency sweep and was approximately $1 \mathrm{~mm}$ in our experiment.

We thank X. Zhao for experimental assistance with the electronics. This project was sponsored by National Institutes of Health grants R29 CA68562 and R01 CA71980.

\section{References}

1. R. R. Alfano and J. G. Fujimoto, eds., Advances in Optical Imaging and Photon Migration, Vol. 2 of Topics in Optics and Photonics Series (Optical Society of America, Washington, D.C., 1996).

2. L.-H. Wang, S. L. Jacques, and X.-M. Zhao, Opt. Lett. 20, 629 (1995).

3. M. Kempe, M. Larionov, D. Zaslavsky, and A. Z. Genack, J. Opt. Soc. Am. A 14, 1151 (1997).

4. L.-H. Wang and X.-M. Zhao, Appl. Opt. 36, 7277 (1997).

5. A. Oraevsky, R. Esenaliev, F. K. Tittel, M. R. Ostermeyer, L.-H. Wang, and S. L. Jacques, Proc. SPIE 2681, 277 (1996).

6. R. A. Kruger, P. Liu, Y. R. Fang, and C. R. Appledorn, Med. Phys. 22, 1605 (1995).

7. F. A. Marks, H. W. Tomlinson, and G. W. Brooksby, Proc. SPIE 1888, 500 (1993).

8. W. Leutz and G. Maret, Physica B 204, 14 (1995). 